\title{
Os efeitos do exercício físico sobre o manejo da dor em pacientes com osteoartrose de joelho: Uma revisão sistemática com meta-análise*
}

\section{The Effects of Physical Exercise on Pain Management in Patients with Knee Oseoarthritis: A Systematic Review with Methanalysis}

\author{
Thiago Casali Rocha1임 Plínio dos Santos Ramos ${ }^{1}$ \\ ${ }^{1}$ Faculdade de Ciências Médicas e da Saúde de Juiz de Fora, Hospital e \\ Maternidade Therezinha de Jesus, Juiz de Fora, MG, Brasil \\ 2 Programa de Pós-Graduação Latu Senso em Fisioterapia Traumato \\ Ortopédica, da Faculdade de Ciências Médicas e da Saúde de Juiz de \\ Fora, Juiz de Fora, MG, Brasil
}

Alessandra Germano Dias ${ }^{2}$ Elaine Angélica Martins ${ }^{2}$

Endereço para correspondência Thiago Casali Rocha, Master, Faculdade de Ciências Médicas e da Saúde de Juiz de Fora, Hospital e Maternidade Therezinha de Jesus, Alameda Salvaterra, 200, Salvaterra, Juiz de Fora, MG, 36033-003, Brasil (e-mail: thiagocasali@physio10.com.br).

Rev Bras Ortop 2020;55(5):509-517.

\section{Resumo}

\section{Palavras-chave \\ - dor \\ - força muscular \\ - osteoartrite de joelho \\ - técnicas de exercício e de movimento}

Objetivo O presente estudo verificou por meio de uma revisão sistemática com metanálise os efeitos de um programa de reabilitação, através de um programa de treinamento físico, para o tratamento da dor e força muscular na osteoartrose (OA) de joelho.

Métodos Foram analisados os estudos publicados entre 2008 e 2018, tendo como referência a base de dados Medline (National Library of Medicine), da qual foram selecionados 7 ensaios clínicos controlados randomizados que pontuaram acima de 8 na escala Physiotherapy Evidence Database (PeDro, na sigla em inglês), sobre programas de exercícios na melhora da dor e força muscular em paciente com OA de joelho. Foi usada a sistematização Preferred Reporting Items for Systematic Reviews and Meta-Analyses (PRISMA, na sigla em inglês) para a elaboração desta revisão e a realização de uma metanálise com o objetivo de evidenciar matematicamente os resultados do exercício físico sobre a dor.

Resultados Os estudos incluídos na análise continham um total de 934 participantes com idade entre 40 a 73 anos, sendo que $34,90 \%$ desses eram do sexo masculino. A maioria dos conjuntos de exercícios oferecidos no tratamento da OA tiveram resultado significativamente positivo em ambos os quesitos, mas principalmente para o alívio da dor (estatisticamente significativo $p<0,003$ ).

Conclusão Inferimos que houve uma melhora da dor em todos os artigos que realizaram fortalecimento muscular, porém ainda há um óbice sobre os protocolos utilizados.

\footnotetext{
Trabalho desenvolvido na Faculdade de Ciências Médicas e da Saúde de Juiz de Fora, Hospital e Maternidade Therezinha de Jesus, Juiz de Fora, MG, Brasil.
}

recebido

04 de Novembro de 2018 aceito

12 de Março de 2019
DOI https://doi.org/ 10.1055/s-0039-1696681. ISSN 0102-3616.
Copyright $\odot 2020$ by Sociedade Brasileira License terms de Ortopedia e Traumatologia. Published by Thieme Revnter Publicações Ltda, Rio de Janeiro, Brazil 


Abstract

Objective The present study verified through a systematic review with meta-analysis the effects of a rehabilitation program, through a physical training program, for the treatment of pain and muscle strength in knee OA.

Methods We analyzed the studies published between 2008 and 2018, with reference to the Medline (National Library of Medicine) database where seven randomized controlled clinical trials were selected that scored above eight on the Physiotherapy Evidence Database (PeDro) scale, on exercise programs to improve pain and muscle strength in a patient with knee OA. The Preferred Reporting Items for Systematic Reviews and Meta-Analyses (PRISMA) systematization was used for the preparation of this review and a meta-analysis was carried out in order to mathematically evidence the results of physical exercise on pain.

Results The studies included in the analysis contained a total of 934 participants aged 40 to 73 years, with $34.90 \%$ of them being male. Most of the exercise sets offered in OA treatment had a significantly positive result in both, but mainly for pain relief (statistically significant $p<0.003$ ).

Conclusion We infer that there was an improvement of pain in all articles that performed muscle strengthening, but there is still an obstacle to the protocols used.

\section{Introdução}

O envelhecimento da população tem crescido em todo o mundo, e, com isso, os desafios da saúde também aumentam, principalmente, no que diz respeito à grande quantidade de doenças que acometem os idosos. Dentre elas, destaca-se a osteoartrose (OA). ${ }^{1}$ Segundo dados de um estudo de Framingham, a OA é a segunda causa de afastamento do trabalho e a principal causa de incapacidade nos idosos. $^{2}$

A OA é descrita como uma doença articular degenerativa e progressiva, não-inflamatória, que consiste na degradação da cartilagem articular e alterações no osso subcondral. ${ }^{3}$ Essa afecção afeta o movimento sincrônico normal da articulação, podendo gerar dor, rigidez, déficit de força muscular e instabilidade articular, sendo que essas condições podem reduzir a mobilidade funcional do indivíduo, levando a perda de função. ${ }^{4}$ Porém, acredita-se que a doença não resulta do processo de envelhecimento, mas de mudanças bioquímicas e estresses biomecânicos que afetam a cartilagem articular. ${ }^{5,6}$

Devido a este fato, sabe-se que a OA de joelho atinge em maior parte o sexo feminino, visto que, anatomicamente a cartilagem dessa região é menos espessa em indivíduos do sexo feminino, apresentando menor área e menor volume, resultando em um aumento da força de cisalhamento no local. ${ }^{7}$

Nguyen et al. ${ }^{8}$ evidenciaram, em um estudo controlado randomizado, que a terapia de exercícios é um tratamento eficaz na melhoria do desempenho físico de pacientes com OA de joelho e comorbidades severas. Em consonância com esta informação, podemos destacar a atualização das diretrizes de 2014 conduzida pela Sociedade Internacional de Osteoartrite (OARSI, na sigla em inglês), que considera a reabilitação o principal tratamento para OA. Através de um estudo de metanálise, concluíram que a terapia de exercícios associada ao treinamento de força e à atividade aeróbica reduzia a dor e melhorava a função física do indivíduo., ${ }^{8,9}$

Lange et al. ${ }^{10}$ avaliaram em uma revisão sistemática a eficácia de um treinamento de resistência para tratar a OA de joelho. Foi observada melhora geral dos sintomas e do desempenho físico dos pacientes, e puderam verificar que mais da metade dos estudos incluídos relataram sucesso na aplicação do treinamento de resistência como: a função física e a força muscular, que melhoraram significativamente quando comparados aos grupos que receberam apenas os cuidados habituais. ${ }^{9,10}$

Tanakar et al., ${ }^{11}$ em uma revisão sistemática com metanálise de ensaios clínicos randomizados, apontam que exercícios aeróbicos associados a exercícios de fortalecimento sem descarga de peso são mais eficazes no alívio da dor em atividades a curto prazo. No entanto, encontra-se na literatura evidência de exercícios de equilíbrio, treinamentos de resistência e exercícios aeróbicos associados à uma redução do quadro álgico, alívio da rigidez e melhoria da função física em pacientes com OA de joelho. ${ }^{12-15}$

Diante dessas circunstâncias surge a necessidade de entender a eficácia do treinamento de exercícios sobre os fatores impactantes da OA de joelho. Há muitas evidências ${ }^{12,13,16,17}$ sobre os benefícios de alguns métodos de reabilitação da OA de joelho, porém o regime ideal de tratamento específico para cada umas das condições ainda é uma dúvida. Dentre esses métodos de reabilitação, encontrase o treinamento físico, que consiste em um método ou programas de exercício físico que podem ser usadas para promover, manter ou restaurar o bem-estar físico e fisiológico de um indivíduo. ${ }^{18}$

Desta forma, o objetivo desse estudo é verificar estudos controlados e randomizados, por meio de uma revisão sistemática dos efeitos de um programa de reabilitação, através de um programa de treinamento físico, para o tratamento da dor e força muscular na OA de joelho. 


\section{Métodos}

Foram incluídos em nossa análise os mais relevantes estudos publicados originalmente na língua inglesa nas bases de dados National Library of Medicine (MEDLINE), Scientific Electronic Library Online (SciELO) e Literatura Latino-Americana e do Caribe em Ciência da Saúde (LILACS), entre janeiro de 2008 e dezembro 2018. Com o objetivo de selecionar os estudos de maior evidência científica, utilizamos para a revisão bibliográfica, somente ensaios clínicos controlados e randomizados (ECCR). Para a busca dos artigos científicos, empregamos as seguintes combinações de palavras-chave: "movement techniques exercise", knee osteoarthritis AND pain AND "muscle strength", AND proprioception trainingbem como suas variações consultadas no Medical Subject Headings (MeSH).

Os critérios de inclusão e exclusão aplicados estão expostos no -Quadro 1.

Os estudos foram selecionados por dois revisores independentes, excluindo aqueles que não estavam relacionados com o tema da revisão e os que pudessem apresentar algum tipo de viés. Para verificar a validade de estudos randomizados elegíveis, pares de revisores trabalhando independentemente e com confiabilidade adequada determinaram a adequação da randomização em relação às condutas de exercícios adotados para os grupos controle e intervenção.

Posteriormente, os resumos dos títulos selecionados foram analisados para identificar aqueles que atendiam aos critérios de inclusão e exclusão. Foram excluídos artigos que não possuíam nenhum dos desfechos do estudo, que não abordam a $\mathrm{OA}$ de joelho, que não possuem um grupo específico ou não realizam nenhum tipo de exercício, e aqueles que eram protocolos de estudo.

Os artigos relevantes foram para avaliação final por meio da pontuação atingida na escala PEDro, com o objetivo de auxiliar os pesquisadores a identificarem rapidamente quais dos estudos selecionados poderão ter validade interna e conter suficiente informação estatística para que os seus resultados possam ser interpretados. Para elegibilidade do

Quadro 1 Critérios de inclusão e exclusão

\begin{tabular}{|l|}
\hline Critérios de inclusão \\
\hline Delineamento: ensaios clínicos controlados e randomizados \\
\hline $\begin{array}{l}\text { Intervenção: programas de exercícios para melhora da dor e } \\
\text { força muscular em paciente com osteoartrose de joelho }\end{array}$ \\
\hline Somente em humanos \\
\hline Idioma: língua inglesa \\
\hline Estudos realizados nos últimos 10 anos. \\
\hline Critérios de exclusão \\
\hline Intervenção: pouco claras, mal descritas ou inadequadas. \\
\hline Forma de publicação: somente resumos \\
\hline Principais variáveis analisadas \\
\hline Dor no joelho \\
\hline Força muscular \\
\hline
\end{tabular}

estudo selecionado para esta revisão sistemática foi necessário pontuar, no mínimo, oito na escala Physiotherapy Evidence Database (PEDro). Além desta escala, utilizamos a escala proposta por Jadad et al. ${ }^{19}$ Esta escala consiste em 5 critérios, e varia de 0 a 5 pontos, na qual o escore menor que 3 indica que o estudo possui baixa qualidade metodológica e, dificilmente, seus resultados poderão ser extrapolados para outros cenários.

\section{Análise estatística}

Para os dados encontrados referentes aos programas de exercício e ao sintoma de dor no joelho, foi realizada a meta-análise com o programa Medcalc 15.8 (BVBA, Ostend, Belgium). A estatística g de Hedges foi utilizada como uma formulação para a diferença média padronizada sob o modelo de efeitos fixos. Em seguida, a estatística de heterogeneidade é incorporada para calcular a diferença média padronizada resumida sob o modelo de efeitos aleatórios utilizando a análise estatística de efeito aleatório e efeito fixo, considerando a heterogeneidade dos estudos. 0 intervalo de confiança de 95\% (IC 95\%) foi calculado para cada estudo individualmente e em seguida, para a combinação dos estudos selecionados. Foram identificados a média e o desvio-padrão de cada estudo e adotados somente os valores de $p<0,05$ como significantes.

\section{Resultados}

Baseados nas palavras-chave descritas anteriormente, foram selecionados 986 artigos nas bases de dados MedLine, Lilacs e ScieLo. Após a aplicação de todos os critérios de inclusão e exclusão, restaram 35 artigos que foram lidos na íntegra e avaliados utilizando a escala PEDro e a escala Jadad, sendo 7 desses os que foram considerados relevantes para revisão sistemática, como é possível observar no fluxograma abaixo (-Figura 1).

As - Tabelas 1 e $\mathbf{2}$ apresentam a pontuação dos 7 estudos utilizados nesta revisão sistemática avaliados pelas escalas PEDro e Jadad, sendo a pontuação mínima de 8 pontos em 11 e 3 pontos em 5 respectivamente.

Os estudos incluídos na análise continham um total de 934 participantes com idades entre 40 e 73 anos, sendo que $34,90 \%$ desses eram do sexo masculino. Os estudos avaliaram a eficácia de um conjunto de exercícios para tratamento da OA de joelho, sendo que alguns compararam ainda os exercícios com outras técnicas de tratamento, que aconteceram em um período médio de 12 semanas. As variáveis analisadas foram dor e força muscular, sendo possível observar que a maioria dos conjuntos de exercícios oferecidos no tratamento da OA tiveram resultado significativamente positivo em ambos os quesitos, mas principalmente no manejo da dor, como mostra a - Tabela 3.

\section{Meta-análise}

Apenas cinco dos sete estudos incluídos nesta revisão forneceram dados suficientes para analisar a dor no joelho após o programa de exercícios. A meta-análise foi realizada com base nos cinco artigos referidos, totalizando uma amostra 
512 Efeitos do exercício físico sobre o manejo da dor em pacientes com osteoartrose de joelho Rocha et al.

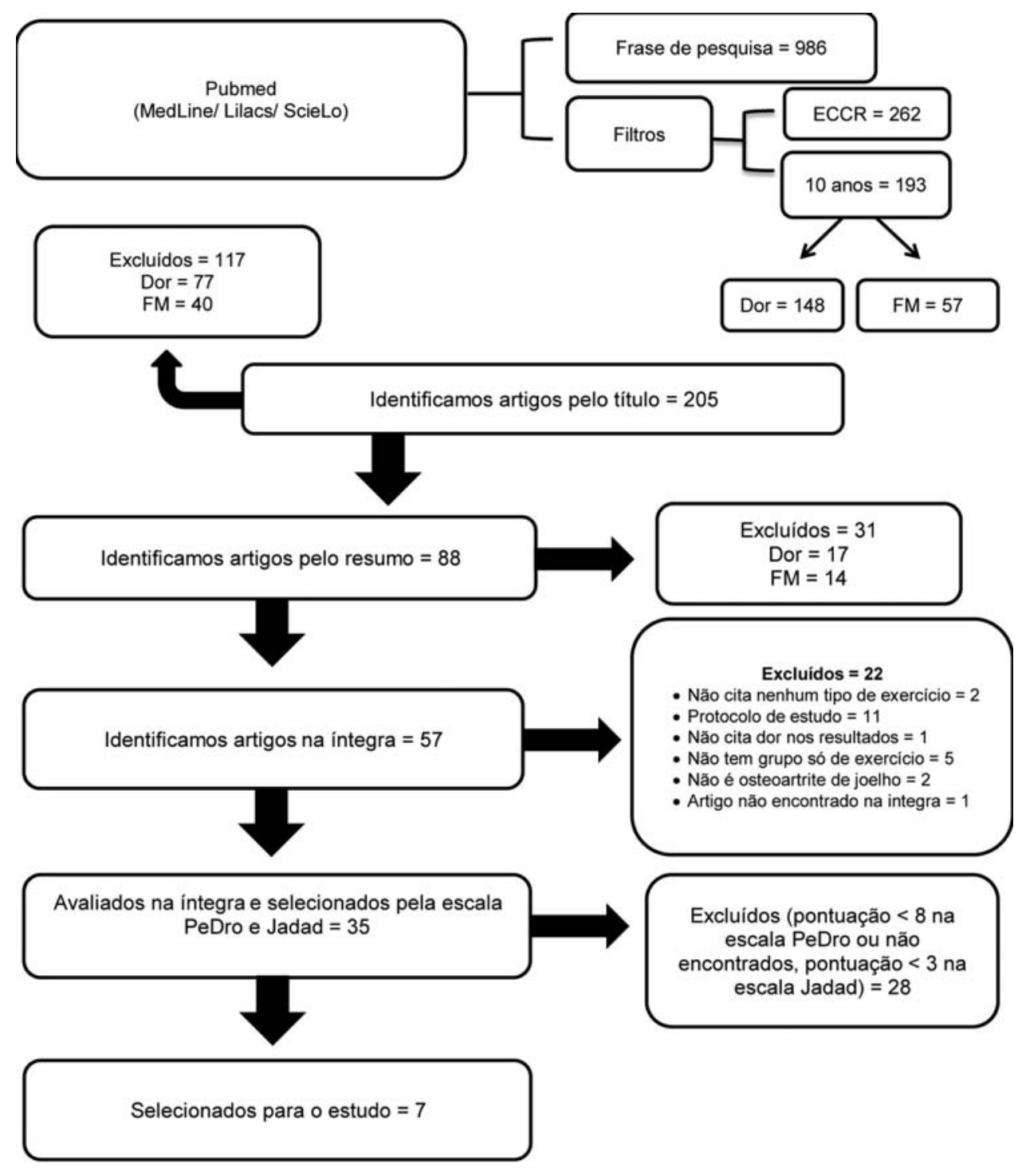

FM: Força Muscular; ECCR: Ensaio Clínico Controlado Randomizado

Fig. 1 Fluxograma.

Tabela 1 Escala Physiotherapy Evidence Database

\begin{tabular}{|c|c|c|c|c|c|c|c|c|c|c|c|c|}
\hline \multicolumn{13}{|l|}{ Escala PEDro } \\
\hline Estudo & C1 & $\mathrm{C} 2$ & $\mathrm{C} 3$ & C4 & C5 & C6 & C7 & $\mathrm{C} 8$ & C9 & C10 & C11 & Score \\
\hline Boon-Whatt Lim et al., $2008^{33}$ & 1 & 1 & 1 & 1 & 0 & 0 & 1 & 1 & 1 & 1 & 1 & $8 / 10$ \\
\hline K.L. Bennell et al., $2010^{25}$ & 1 & 1 & 1 & 1 & 0 & 0 & 1 & 1 & 1 & 1 & 1 & $8 / 10$ \\
\hline G. Kelley Fitzgerald et al., $2011^{20}$ & 1 & 1 & 1 & 1 & 1 & 0 & 1 & 0 & 1 & 1 & 1 & $8 / 10$ \\
\hline Saccomanno MF et al., $2016^{21}$ & 1 & 1 & 1 & 1 & 0 & 0 & 1 & 1 & 1 & 1 & 1 & $8 / 10$ \\
\hline Palmer S et al., $2014^{22}$ & 1 & 1 & 1 & 1 & 1 & 0 & 1 & 0 & 1 & 1 & 1 & $8 / 10$ \\
\hline Holsgaard-Larsen A et al., $2017^{23}$ & 1 & 1 & 1 & 1 & 0 & 0 & 1 & 1 & 1 & 1 & 1 & $8 / 10$ \\
\hline Jorge RT et al., $2015^{24}$ & 1 & 1 & 1 & 1 & 0 & 0 & 1 & 1 & 1 & 1 & 1 & $8 / 10$ \\
\hline
\end{tabular}

Abreviatura: PEDro, Physiotherapy Evidence Database.

com 520 voluntários. Entre os resultados relacionados, cinco dos sete artigos utilizaram como método de avaliação da dor o questionário Western Ontario and McMaster Universities Arthritis Index (WOMAC), e, dentre eles, dois demostraram que o exercício é efetivo quando comparado com outras técnicas de tratamento da OA de joelho, como é possível observar na - Figura 2 e na - Tabela 4 . O gráfico em florestplot evidencia a análise do efeito do programa de exercícios 
Tabela 2 Escala de Jadad

\begin{tabular}{|c|c|c|c|c|c|c|c|}
\hline Itens & $\begin{array}{l}\text { Boon-Whatt } \\
\text { Lim et al., } \\
200832\end{array}$ & $\begin{array}{l}\text { K.L. Bennell } \\
\text { et al., } \\
2010^{24}\end{array}$ & $\begin{array}{l}\text { G. Kelley } \\
\text { Fitzgerald } \\
\text { et al., } 201119\end{array}$ & $\begin{array}{l}\text { Sacco manno } \\
\text { MF et al., } \\
2016^{20}\end{array}$ & $\begin{array}{l}\text { Palmer S } \\
\text { et al., } \\
2014^{21}\end{array}$ & $\begin{array}{l}\text { Holsgaard- } \\
\text { Larsen A et al., } \\
2017^{22}\end{array}$ & $\begin{array}{l}\text { Jorge RT } \\
\text { et al., } \\
2015^{23}\end{array}$ \\
\hline $\begin{array}{l}\text { O estudo foi descrito } \\
\text { como randomizado? }\end{array}$ & 1 & 1 & 1 & 1 & 1 & 1 & 1 \\
\hline $\begin{array}{l}\text { A randomização foi } \\
\text { descrita e é adequada? }\end{array}$ & 1 & 1 & 1 & 1 & 1 & 1 & 1 \\
\hline $\begin{array}{l}\text { Houve comparações } \\
\text { e resultados? }\end{array}$ & 1 & 1 & 1 & 1 & 1 & 1 & 1 \\
\hline $\begin{array}{l}\text { As comparações } \\
\text { e resultados } \\
\text { foram descritos } \\
\text { e são adequados? }\end{array}$ & 1 & 1 & 1 & 1 & 1 & 1 & 1 \\
\hline $\begin{array}{l}\text { Foram descritas } \\
\text { perdas e exclusões? }\end{array}$ & 1 & 1 & 1 & 1 & 1 & 1 & 1 \\
\hline TOTAL & 5 & 5 & 5 & 5 & 5 & 5 & 5 \\
\hline
\end{tabular}

sobre a dor na região do joelho. Os resultados da esquerda indicam os valores favoráveis à influência do programa de exercícios para a diminuição do quadro álgico quando comparado com o grupo controle, sendo o efeito combinado representado pelo losango, que evidenciou uma diferença estatisticamente significativa $(p=0,0031)$.

\section{Discussão}

Este estudo teve como objetivo analisar a eficácia de um grupo de exercícios no tratamento da OA de joelho, sendo a dor e a força muscular os principais desfechos abordados. Nossos resultados demonstram que um programa de reabilitação que inclua o fortalecimento de um determinado grupamento muscular tem efeitos positivos sobre a dor.

Os músculos quadríceps femoral, ísquio crural, psoas maior, glúteo máximo e médio, gastrocnêmios, tensor da fáscia lata, adutor longo, adutor curto, grácil, adutor magno, e sartório são os mais evidenciados nesta revisão sistemática. ${ }^{20-25}$

Dentre os músculos citados nos programas de reabilitação, o foco principal está no quadríceps femoral. O fortalecimento deste é comumente indicado no tratamento e progressão da $\mathrm{OA}$, visto que esse possui efeito condroprotetor estático e dinâmico da articulação do joelho, e sua fraqueza pode gerar sobrecarga articular causando dor e instabilidade. ${ }^{26}$ Segundo O'Reilly et al., ${ }^{27}$ existe uma relação inversa entre a força muscular do quadríceps, em pacientes com OA, e o relato de dor, ou seja, quanto maior a força muscular menor a dor. ${ }^{27,28}$ Porém, o músculo em sua maior potência, pode aumentar a força de cisalhamento, ou a força compressiva, dentro da articulação podendo aumentar a dor no joelho acometido pela $\mathrm{OA}$, com isso, quando a articulação está em um posição mais fletida o músculo fica mais relaxado e essas forças diminuem sobre a articulação gerando um maior conforto ao paciente, reduzindo assim, a ativação muscular. ${ }^{29,30} \mathrm{Na}$ literatura ainda há discussões quanto à relação entre quadríceps femoral e OA de joelho, pois existem controvérsias se a fraqueza muscular contribui para a patologia ou a patologia causa a fraqueza muscular. ${ }^{31,32}$
O desequilíbrio artrocinemático causado por fatores mecânicos pode também ter influência na causa e na progressão da OA do joelho, pois o alinhamento desempenha um papel importante que determina a distribuição da carga através dessa articulação, minimizando o efeito do impacto, de modo que pode ser hipotetizado que o aumento da força muscular é uma das principais causas de impacto, redução da dor e incapacidade. Surge então, a necessidade de se entender sobre a influência do mau alinhamento articular para tratar a OA. O estudo de Lim et al., ${ }^{33}$ mostrou que o fortalecimento do quadríceps em um grupo de pacientes com desalinhamento grave no joelho originou a piora da dor em relação ao grupo controle, ao contrário do grupo que apresentava um alinhamento melhor. Além disso, ele verifica que o fortalecimento do quadríceps não melhora o alinhamento do joelho, mostrando que com um aumento de $3 \%$ na força do quadríceps o desalinhamento no joelho piora em 1 grau. Este achado não necessariamente vai de encontro com os nossos resultados, porém ressalta a importância de se observar o alinhamento articular antes de se iniciar um trabalho de fortalecimento, para que de fato se tenha resultados positivos sobre os desfechos, principalmente em relação à dor. ${ }^{34}$

A amplitude de movimento também é um quesito importante sobre a eficácia de um tratamento articular, pois pacientes com OA crônica do joelho podem apresentar encurtamento quando submetidos à imobilização ou inatividade devido à dor, resultando em contraturas de cápsulas articulares e encurtamento adaptativo. ${ }^{35}$ Partindo do pressuposto que músculos mais alongados possuem maior torque, os benefícios dos programas de alongamento estão além do alinhamento e equilíbrio muscular. Com isso a terapia de alongamento como um tratamento adjuvante se tornaria uma técnica favorável de se incluir em um programa de exercícios para o tratamento de OA. Tanto a facilitação neuromuscular proprioceptiva (FNP) como o alongamento estático mostram ótimos resultados, porém o estiramento do FNP foi mais efetivo que o exercício de alongamento estático. ${ }^{35,36}$ Apenas três dos artigos incluídos na presente revisão apresentam em seu programa exercícios de alongamento/estiramento. ${ }^{21,23,33}$ 


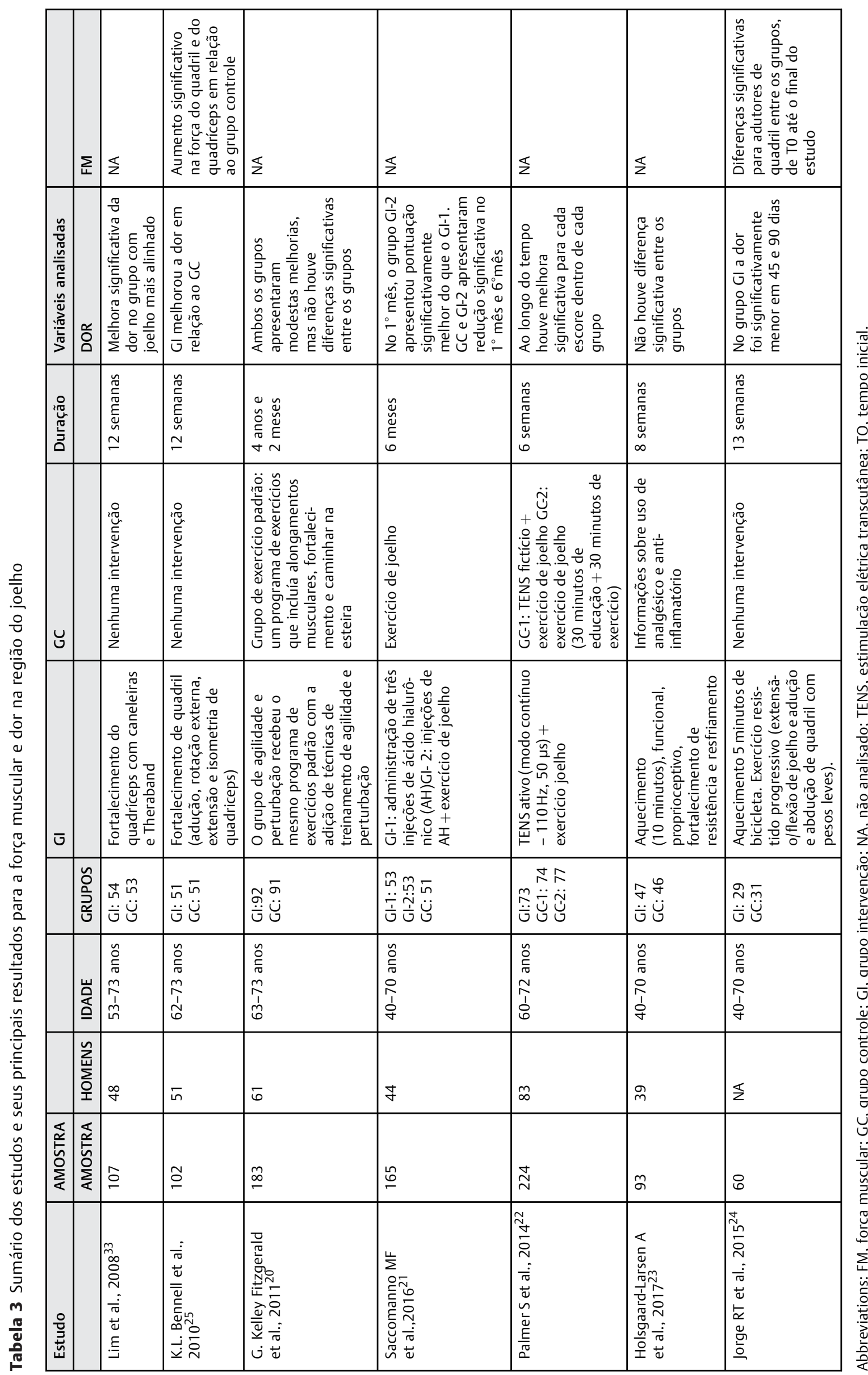




\section{Metanalise}

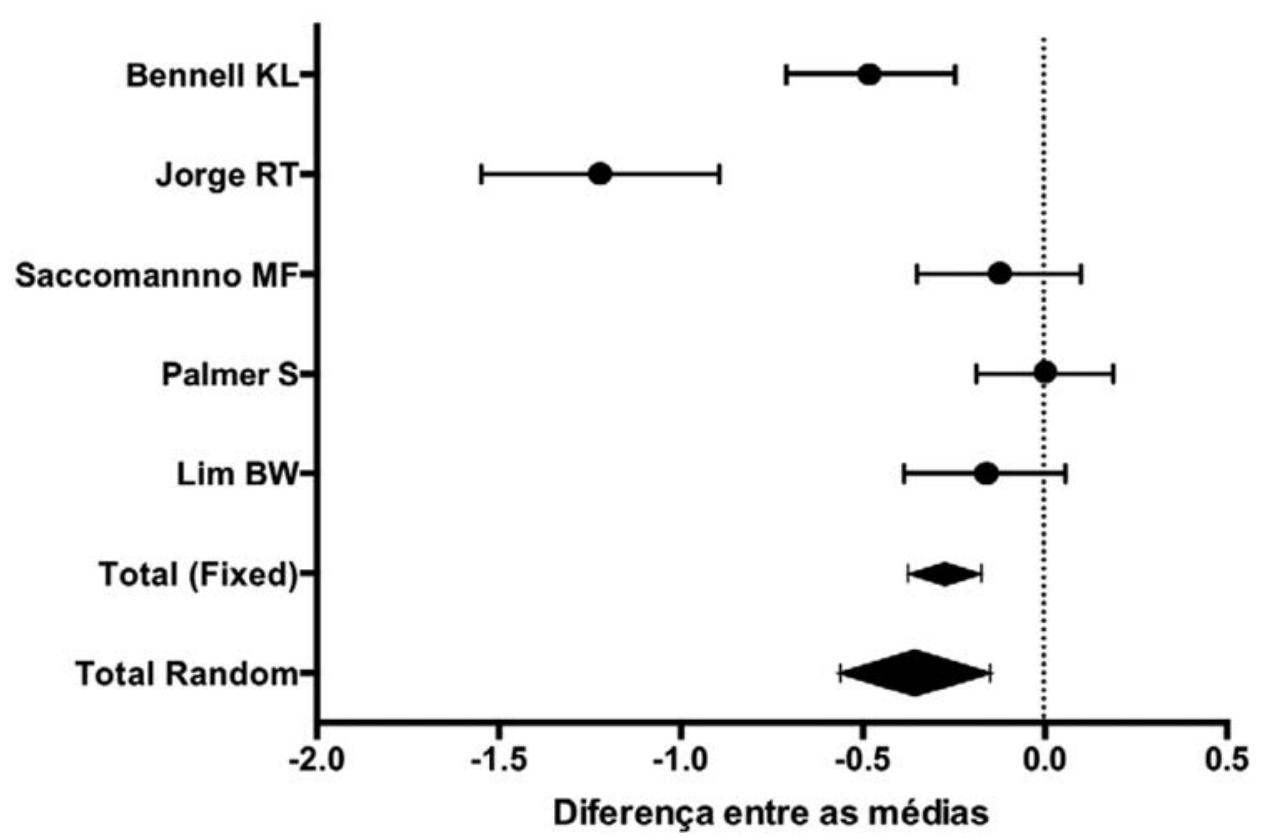

Fig. 2 Gráfico Forest Plot dos estudos incluídos na análise do efeito fixo e aleatório, diferença da média padronizada adotando intervalo de confiança $95 \%$.

Tabela 4 Dados da metanálise

\begin{tabular}{|l|l|l|l|l|l|}
\hline Estudo & N1 & N2 & TOTAL & SMD & 95\% IC \\
\hline Lim et al., 2008 & 54 & 53 & 107 & $-0,165$ & $-0,549$ to 0,219 \\
\hline Bennell et al., 2010 & 51 & 51 & 102 & $-0,481$ & $-0,880$ to $-0,0824$ \\
\hline Jorge et al., 2015 & 29 & 31 & 60 & $-0,122$ & $-1,788$ to $-0,655$ \\
\hline Saccomanno et al., 2016 & 51 & 53 & 104 & $-0,126$ & $-0,516$ to 0,263 \\
\hline Palmer et al., 2014 & 73 & 74 & 147 & 0,000 & $-0,326$ to 0,326 \\
\hline Total (Efeito fixo) & 258 & 262 & 520 & $-0,275$ & $-0,450$ to $-0,101$ \\
\hline Total (Efeito randomizado) & 258 & 262 & 520 & $-0,357$ & $-0,712$ to $-0,00181$ \\
\hline Nível de significância & $P=0,0031$ & & & \\
\hline
\end{tabular}

Abbreviations: IC, intervalo de confiança; N1, amostra do grupo intervenção; N2, amostra do grupo controle; SMD, diferença entre as medias.

A maioria dos estudos incluídos nessa revisão aborda além do fortalecimento a adição de técnicas de equilíbrio e propriocepção. Porém, apenas o estudo de Fitzgerald et al. ${ }^{20}$ avaliou separadamente essas técnicas, não encontrando evidências significativas de que esses exercícios melhoram a dor e força muscular em pacientes com OA, esse achado não corrobora com o estudo de Diracoglu et al., ${ }^{37}$ que aplicou exercícios de cinestesia e equilíbrio em um grupo de mulheres com OA de joelho em comparação com um grupo que recebeu apenas fortalecimento, obtendo resultado positivo na força muscular, na qualidade de vida e a escala de função física do questionário de WOMAC.

Para mensurar cada um desses desfechos que escolhemos, os artigos optaram em sua totalidade por usar instrumentos de avaliação como o questionário WOMAC e a escala visual analógica (EVA). O questionário WOMAC é um instrumento de fácil aplicação, com baixo custo e específico para OA de joelho, composto por três domínios, dor, rigidez e funcionalidade. ${ }^{38}$ Já a EVA é um instrumento numérico, de 0 a 10, com $10 \mathrm{~cm}$ de comprimento, onde a sensação dolorosa é validada, quanto maior a numeração maior o nível da dor. ${ }^{30}$

Confrontando os pontos de cada instrumento de avaliação podemos observar que dentro da necessidade de avaliar um quesito especifico, como no caso da OA, a escala EVA e o questionário WOMAC se tornam muito subjetivos, porém este tem como finalidade especificar a dor momentânea somente na $\mathrm{OA}$ de joelho, sendo o mais satisfatório nesse tipo de avaliação. ${ }^{30,38}$

Com base no que há em comum nas evidências utilizadas, concluímos que, para que um programa de reabilitação seja benéfico no tratamento da OA ele deve avaliar satisfatoriamente o desalinhamento articular do joelho, e posteriormente desenvolver um plano de tratamento adequado às necessidades de cada paciente. Baseado nessa avaliação, o terapeuta se 
guiará para formular um programa de treinamento físico com enfoque no grupamento muscular adequado.

O ideal, segundo os nossos resultados, seriam programas que possuíssem exercícios com enfoque no fortalecimento isométrico e isotônico dos músculos quadríceps femoral e ísquios crurais, sendo o isotônico de quadríceps mais importante, alongamento dinâmico (PNF) dos músculos ísquios crurais e gastrocnêmios, além de exercícios de propriocepção e equilíbrio.

Os estudos analisados apresentam algumas limitações, dentre elas a falta de descrição detalhada das intervenções, assim como a falta de descrição da carga utilizada e a evolução dos exercícios, principalmente em relação aos alongamentos musculares, prejudicando na elaboração final de um programa de treinamento físico adequado. A duração da intervenção possuiu uma grande variação não os permitindo alcançar um consenso sobre o tempo ideal de tratamento da OA. Os benefícios a longo prazo de exercícios para terapia e possível prevenção de $\mathrm{OA}$ ainda não são conhecidos, devido à escassez de estudos sobre esses efeitos.

Outra limitação observada é a ausência de um instrumento padrão-ouro para a avaliação da força muscular, como o uso de um dinamômetro, comprometendo a análise quantitativa desta variável. A hipótese de que possa ser o pela disponibilidade reduzida e custo elevado dos aparelhos para a avaliação.

\section{Conclusão}

Frente aos resultados encontrados nessa análise, inferimos que houve uma melhora da dor em todos os artigos que realizaram fortalecimento muscular; porém, os protocolos utilizados não foram devidamente descritos, dificultando um padrão para elaborar um programa de exercícios físicos específicos para o tratamento da OA de joelho. Em adendo, apenas dois artigos avaliaram a força muscular, provavelmente pela dificuldade de medida/utilização do instrumento adequado.

\section{Conflito de Interesses}

Os autores declaram não haver conflito de interesses.

\section{Referências}

1 van der Pas S, Castell MV, Cooper C, et al. European project on osteoarthritis: design of a six-cohort study on the personal and societal burden of osteoarthritis in an older European population. BMC Musculoskelet Disord 2013;14:138

2 Felson DT. The epidemiology of knee osteoarthritis: results from the Framingham Osteoarthritis Study. Semin Arthritis Rheum 1990;20(03, Suppl 1):42-50

3 Lane NE, Brandt K, Hawker G, et al. OARSI-FDA initiative: defining the disease state of osteoarthritis. Osteoarthritis Cartilage 2011; 19(05):478-482

4 Conn VS, Hafdahl AR, Minor MA, Nielsen PJ. Physical activity interventions among adults with arthritis: meta-analysis of outcomes. Semin Arthritis Rheum 2008;37(05):307-316

5 Kerkhof HJ, Bierma-Zeinstra SM, Arden NK, et al. Prediction model for knee osteoarthritis incidence, including clinical, genetic and biochemical risk factors. Ann Rheum Dis 2014;73(12):2116-2121
6 Sharma L, Song J, Dunlop D, et al. Varus and valgus alignment and incident and progressive knee osteoarthritis. Ann Rheum Dis 2010;69(11):1940-1945

7 Otterness IG, Eckstein F. Women have thinner cartilage and smaller joint surfaces than men after adjustment for body height and weight. Osteoarthritis Cartilage 2007;15(06):666-672

8 Nguyen C, Lefèvre-Colau MM, Poiraudeau S, Rannou F. Rehabilitation (exercise and strength training) and osteoarthritis: A critical narrative review. Ann Phys Rehabil Med 2016;59(03): 190-195

9 McAlindon TE, Bannuru RR, Sullivan MC, et al. OARSI guidelines for the non-surgical management of knee osteoarthritis. Osteoarthritis Cartilage 2014;22(03):363-388

10 Lange AK, Vanwanseele B, Fiatarone Singh MA. Strength training for treatment of osteoarthritis of the knee: a systematic review. Arthritis Rheum 2008;59(10):1488-1494

11 Tanaka R, Ozawa J, Kito N, Moriyama H. Efficacy of strengthening or aerobic exercise on pain relief in people with knee osteoarthritis: a systematic review and meta-analysis of randomized controlled trials. Clin Rehabil 2013;27(12):1059-1071

12 Levinger P, Dunn J, Bifera N, Butson M, Elias G, Hill KD. High-speed resistance training and balance training for people with knee osteoarthritis to reduce falls risk: study protocol for a pilot randomized controlled trial. Trials 2017;18(01):384

13 Mat S, Tan MP, Kamaruzzaman SB, Ng CT. Physical therapies for improving balance and reducing falls risk in osteoarthritis of the knee: a systematic review. Age Ageing 2015;44(01):16-24

14 Hatfield GL, Morrison A, Wenman M, Hammond CA, Hunt MA. Clinical Tests of Standing Balance in the Knee Osteoarthritis Population: Systematic Review and Meta-analysis. Phys Ther 2016;96(03):324-337

$15 \mathrm{Li}$ Y, Su Y, Chen S, et al. The effects of resistance exercise in patients with knee osteoarthritis: a systematic review and meta-analysis. Clin Rehabil 2016;30(10):947-959

16 Iwamoto J, Sato Y, Takeda T, Matsumoto H. Effectiveness of exercise for osteoarthritis of the knee: A review of the literature. World J Orthop 2011;2(05):37-42

17 Jan MH, Lin CH, Lin YF, Lin JJ, Lin DH. Effects of weight-bearing versus nonweight-bearing exercise on function, walking speed, and position sense in participants with knee osteoarthritis: a randomized controlled trial. Arch Phys Med Rehabil 2009;90(06): 897-904

18 Bartholdy C, Juhl C, Christensen R, Lund H, Zhang W, Henriksen M. The role of muscle strengthening in exercise therapy for knee osteoarthritis: A systematic review and meta-regression analysis of randomized trials. Semin Arthritis Rheum 2017;47(01):9-21

19 Jadad AR, Moore RA, Carroll D, et al. Assessing the quality of reports of randomized clinical trials: is blinding necessary? Control Clin Trials 1996;17(01):1-12

20 Fitzgerald GK, Piva SR, Gil AB, Wisniewski SR, Oddis CV, Irrgang JJ. Agility and perturbation training techniques in exercise therapy for reducing pain and improving function in people with knee osteoarthritis: a randomized clinical trial. Phys Ther 2011;91(04): 452-469

21 Saccomanno MF, Donati F, Careri S, Bartoli M, Severini G, Milano G. Efficacy of intra-articular hyaluronic acid injections and exercisebased rehabilitation programme, administered as isolated or integrated therapeutic regimens for the treatment of knee osteoarthritis. Knee Surg Sports Traumatol Arthrosc 2016;24 (05):1686-1694

22 Palmer S, Domaille M, Cramp F, et al. Transcutaneous electrical nerve stimulation as an adjunct to education and exercise for knee osteoarthritis: a randomized controlled trial. Arthritis Care Res (Hoboken) 2014;66(03):387-394

23 Holsgaard-Larsen A, Clausen B, Søndergaard J, Christensen R, Andriacchi TP, Roos EM. The effect of instruction in analgesic use compared with neuromuscular exercise on knee-joint load in 
patients with knee osteoarthritis: a randomized, single-blind, controlled trial. Osteoarthritis Cartilage 2017;25(04):470-480

24 Jorge RT, Souza MC, Chiari A, et al. Progressive resistance exercise in women with osteoarthritis of the knee: a randomized controlled trial. Clin Rehabil 2015;29(03):234-243

25 Bennell KL, Hunt MA, Wrigley TV, et al. Hip strengthening reduces symptoms but not knee load in people with medial knee osteoarthritis and varus malalignment: a randomised controlled trial. Osteoarthritis Cartilage 2010;18(05):621-628

26 Creamer P, Lethbridge-Cejku M, Hochberg MC. Factors associated with functional impairment in symptomatic knee osteoarthritis. Rheumatology (Oxford) 2000;39(05):490-496

27 O'Reilly SC, Jones A, Muir KR, Doherty M. Quadriceps weakness in knee osteoarthritis: the effect on pain and disability. Ann Rheum Dis 1998;57(10):588-594

28 Avelar Di Sabatino Santos ML, Fabiano Gomes W, Zille de Queiroz B, Brito Rosa NMd, Sirineu Pereira D, Domingues Dias JM, et al. Desempenho muscular, dor, rigidez e funcionalidade de idosas com osteoartrite de joelho. Acta Ortop Bras 2011;19(04):

29 Schipplein OD, Andriacchi TP. Interaction between active and passive knee stabilizers during level walking. J Orthop Res 1991;9 (01):113-119

30 Bolognese JA, Schnitzer TJ, Ehrich EW. Response relationship of VAS and Likert scales in osteoarthritis efficacy measurement. Osteoarthritis Cartilage 2003;11(07):499-507

31 Alnahdi AH, Zeni JA, Snyder-Mackler L. Muscle impairments in patients with knee osteoarthritis. Sports Health 2012;4(04):284-292
32 Segal NA, Glass NA, Felson DT, et al. Effect of quadriceps strength and proprioception on risk for knee osteoarthritis. Med Sci Sports Exerc 2010;42(11):2081-2088

33 Lim BW, Hinman RS, Wrigley TV, Sharma L, Bennell KL. Does knee malalignment mediate the effects of quadriceps strengthening on knee adduction moment, pain, and function in medial knee osteoarthritis? A randomized controlled trial. Arthritis Rheum 2008;59(07):943-951

34 Foroughi N, Smith RM, Lange AK, Singh MA, Vanwanseele B. Progressive resistance training and dynamic alignment in osteoarthritis: A single-blind randomised controlled trial. Clin Biomech (Bristol, Avon) 2011;26(01):71-77

35 Weng MC, Lee CL, Chen $\mathrm{CH}$, et al. Effects of different stretching techniques on the outcomes of isokinetic exercise in patients with knee osteoarthritis. Kaohsiung J Med Sci 2009;25(06):306-315

36 Ferreira de Meneses SR, Hunter DJ, Young Docko E, Pasqual Marques A. Effect of low-level laser therapy (904 nm) and static stretching in patients with knee osteoarthritis: a protocol of randomised controlled trial. BMC Musculoskelet Disord 2015; $16: 252$

37 Diracoglu D, Aydin R, Baskent A, Celik A. Effects of kinesthesia and balance exercises in knee osteoarthritis. J Clin Rheumatol 2005;11 (06):303-310

38 Marx FC, Oliveira LM, Bellini CG, Ribeiro MCC. Tradução e validação cultural do questionário algofuncional de Lequesne para osteoartrite de joelhos e quadris para a língua portuguesa. Rev Bras Reumatol 2006;46(04):253-260 